\title{
Fraud Prevention in Taxation System of Pakistan Using Blockchain Technology
}

\author{
Daniel Mago Vistro ${ }^{1, *}$ Muhammad Shoaib Farooq², Attique Ur Rehman², Mohsin A. \\ $\mathrm{Khan}^{2}$
}

${ }^{1}$ School of Computing, Asia Pacific University, Kuala lumpur, Malaysia.

${ }^{2}$ School of System and Technology, University of Management and Technology, Pakistan.

*Corresponding author. Email: Daniel.mago@staffemail.apu.edu.my

\begin{abstract}
Taxation department of Pakistan collects and keeps record of legislated taxes imposed by governments. Pakistan is among those countries which still maintain records of taxations in registers, thus maintaining immutability of records is really hard. Tax records have great risk of being lost or altered by someone and results in leading towards committing fraud in taxes. Blockchain has provided advantage to many industries because of its enhanced security and traceability in current trustless environment. We have presented a tax management solution which has an objective to provide secure, efficient and auditable system in Pakistan environment using blockchain technology. Our proposed methodology is about digitizing of the records of taxations using the blockchain distributed ledger based technology. Finally, future work prediction is also given, which will help getting directions for controlling fraud cases from most senior officers posts.
\end{abstract}

Keywords: Blockchain, Distributed, Ledger, Taxation, Technology.

\section{INTRODUCTION}

Taxation department is government authorized department which collects, maintains, manage the taxations which are applied by the government on goods, services, companies, professionals, buildings, businesses etc. Taxes are paid for the purpose of economical development of the country. Tax percentage is different for each category; major categories include firms, goods, societies and individuals [1].

In Pakistan only 5\% of Pakistani people pay indirect taxes and other $95 \%$ of people keep avoiding paying these taxes by not showing their income or by making good relation with any senior tax officer or practitioner [2]. Indirect Tax collection is the most difficult and challenging task to be done in Pakistan from last years. This is due to unprofessional behavior of tax officers and insincerity with their job and country [3].

Most Pakistani people are not much aware of tax system and do the same as their fathers and grandfathers were doing to save themselves from paying tax. People also have lost trust in the government that whether their tax money is going towards development of Pakistan or not, this is also the major cause that people have lost their trust in the government [4].

The Taxation department is lacking through its management. It also has poor management of keeping tax records. Records are being maintained in the copy registers. Traceability of change in registers and maintains immutability is quite challenging in registers. Tax officers maintain tax records of firms and individual in the registers [5].

Security for each transaction will be provided by blockchain [23-26] which will make each tax safe money safe and traceability will gain trust of Pakistani people that their money is going under development of their country. When people will know that the record entered in blockchain will never be changed or erased then they will trust the government officials and feel safe to pay their tax money. Blockchain ecosystem is presented in Figure 1, in which the main features of blockchain are presented. Distributed ledger distributes each transaction or record entry to each member of that blockchain, this will provide immutability. Privacy and Security are those main features for which we are shifting from registers to blockchain technology, because prevention of fraud can 
be only done when there is full proof security, hence these features make the blockchain best to choose for this type of scenario [6] [15-22].

We have proposed a solution for preventing the alteration or deletion of records; we have digitized all the records that were being maintained on copy registers. Only digitization was not perfect solution for this, because only digitization might lead to fraud and such cases again, as records can also be edited and deleted in computer systems or in a normal database too.

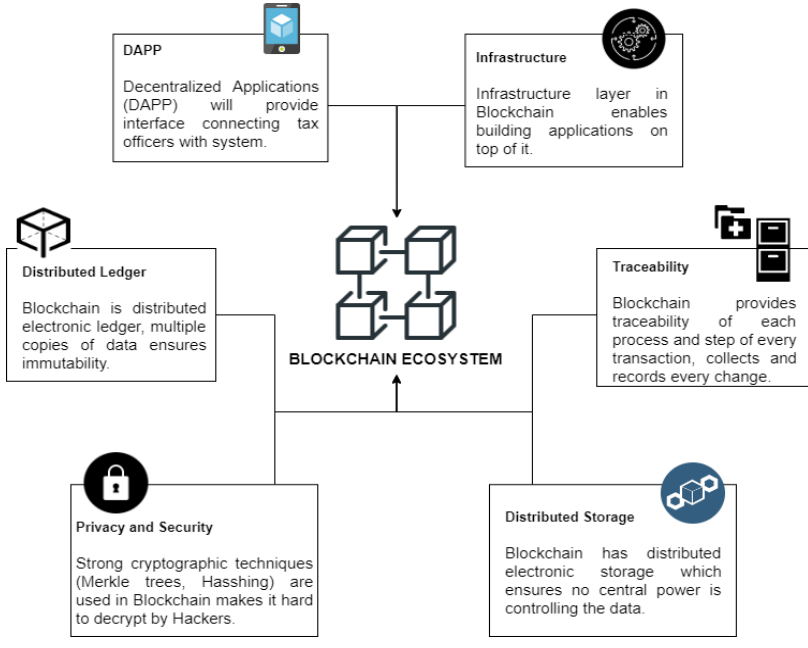

Figure 1 Blockchain Ecosystem.

Blockchain and ledger technologies made every record secure and not able to be edited again ever as they provide so many securities feature that normal database cannot provide [7]. Blockchain decentralization made the records tamperproof which means no tampering of records would be done.

In this research paper, introduction has been discussed in Section I, related work would be discussed in Section II, detailed discussion on proposed methodology/solution will be discussed in Section III, reason for selecting this solution will be discussed in the Section IV. In the end we will conclude the research paper in Section V.

\section{RELATED WORK}

Milla Sepliana [8] used a qualitative approach evaluate blockchain technology that whether they can be applied to Value Added Tax (VAT) system or not. And if they are eligible to be applied then what would be results. This paper was based on the environment and tax system of Indonesia. In Indonesia there are serial numbers of invoices for every tax record which is called Tax Invoice Serial

Number (TISN). It was proposed that if they apply blockchain technology of type permissioned blockchain in TISM system then the system will be more efficient and quicker. In their paper, it was also described that how blockchain based system would be better than conventional system. Each transaction will be tracked and monitore by DGT; DGT in Indonesia is Directorate General of Taxation officer. However, this proposed solution was only f $\neg$ or Tax Invoices but not the whole tax system, there are still chances of fraud from other side of the system.

Frankowski and Barański [9] discussed in their paper that taxes are now being digitized in modern way and into different shapes. Through blockchain technology the way of paying taxes and submitting them into databases has been totally changed. Many countries are noticed that they have implemented this modern way of digitizing taxes in the continent of Europe, these countries include: Brazil and South America. It is proved that this technology has brought better compliance and greater efficiency in tax authorities. The process for paying the taxes became easy and time saving. However, in this paper the architecture or model of system using blockchain being used in Brazil and South America was not clearly defined and explained.

Experts in Tax and Technology [10] wrote a paper which was published on paper water house cooper's platform and discussion was done on that how blockchain technology can bring up improvement in the tax management system. It was as survey paper and suggestions from government law officials and tax officials were taken regarding this implementation on tax system. Transparency, control, security and real-time information, these characteristics can be really helpful in making tax management system really efficient and fraud proof. But there was not example or real time scenarios were discussed here which caused this paper to be bookish kind of paper.

We have proposed a solution for preventing frauds in taxation department of Pakistan using the similar approach as discussed in other related papers we have discussed. But we have applied the blockchain ledger technology to whole tax management system not only on the tickets compared to other papers. In our paper we have showed that how blockchain would show efficiency in Pakistani system and how it should be working.

\section{PROPOSED SOLUTION FOR TAX MANAGMENT}

This research has presented efficient and secure solution to be implemented on Taxation management system for managing the tax records and maintain the log of each action taken in the system. This secure and fraud proof solution helps the country economical condition grow towards development and gain the trust of people of Pakistan to give their tax with no stress that their money could be stolen or misused. 


\subsection{Proposed Framework}

We have proposed high level architecture diagram in Figure 2. Stakeholders of our proposed framework are tax officers and inspectors who are responsible for managing the tax management system and the tax payer, who pay tax according to the tax he has to pay in the bank. Tax management system consists of interplanetary file system (IPFS) which provides peer to peer hypermedia protocol that makes the web safer and faster [11].

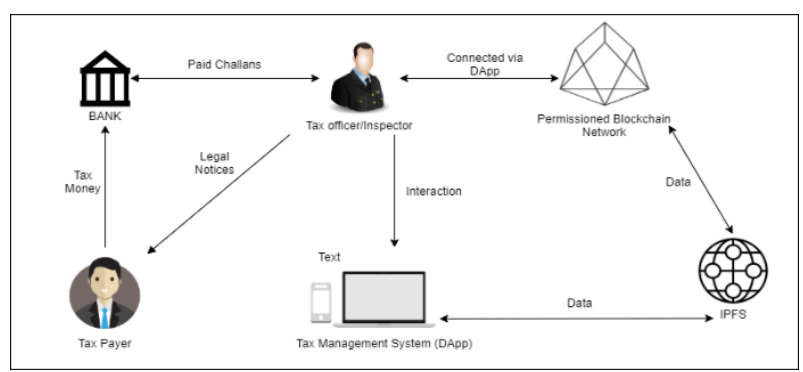

Figure 2 Proposed Framework.

Tax management system is designed as a decentralized application (DAPP) and has blockchain distributed ledger based technology. Our proposed framework describes that when a tax payer pays his tax in the bank, then tax officers or inspectors verifies his payment from the bank and enters the tax payer amount in the tax management system application. Tax officers can also see which person have not paid the tax yet and then they can send legal notices to them. If they don't pay the tax then they will get punishment for that which is defined as per Pakistan's law. In our proposed framework it is shown that permissioned blockchain is used instead permission less blockchain because our system is not publically available to every other user. Only tax officers and inspectors are allowed to access it, not even tax payers are allowed to use it. Our system is to make sure that each tax record is kept safe and not editable for anyone and if someone tries to edit then he would be called for explanations in courts and case would be filed on him.

\subsection{Proposed layered Architecture}

Layered architecture for proposed solution is shown in Figure 3. On interface layer, the applications of taxation management system would be running, decentralized application (DAPP) and web portal for tax officers will take place in application layer. Application layer verifies identification of users and records. Protocol layer provides security by applying consensus algorithms, which makes blockchain database harder to hack.

Permissioned blockchain will also prevent any other user entering into our tax management system. Network layer ensures trusted environment and each node is connected to every other node within the network and no other node is allowed to enter, as it is permissioned blockchain. Infrastructure layer is responsible for storing and management of records and every data in blockchain. This layer is also responsible for sharing data between each other node inside its own permissioned blockchain network. After every transaction, the data is shared to all other nodes.

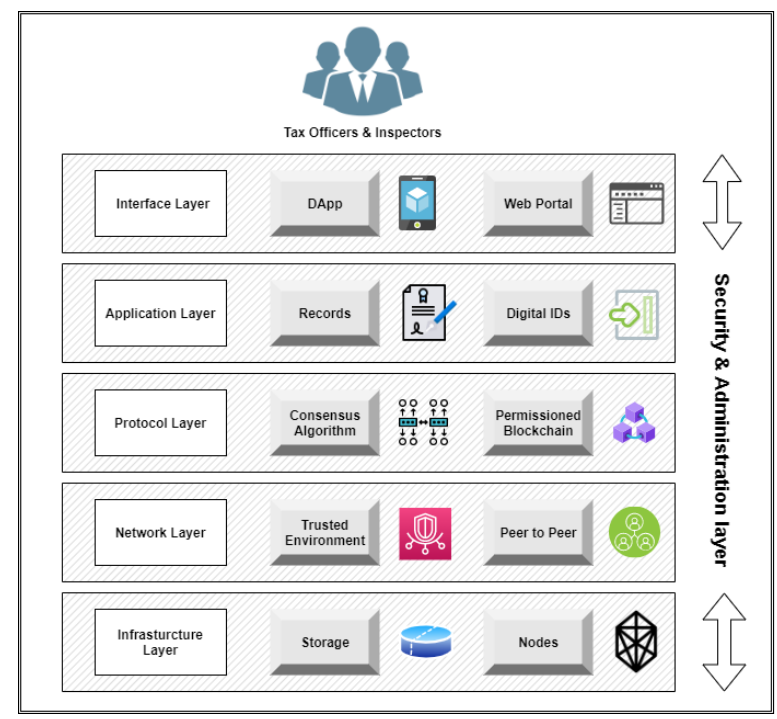

Figure 3 Proposed Blockchain Layered Architecture.

\section{WHY DAPP WHY NOT APP?}

The reason that we are suggesting the application to be built in blockchain based app (Decentralized Applications - DAPP) but not on simple applications (APP) is discussed here. An application is centralized and there is lesser amount of security as compared to which DAPP provides. In APP, people have lost trust to share their information as hackers might use it for harmful purposes [12].

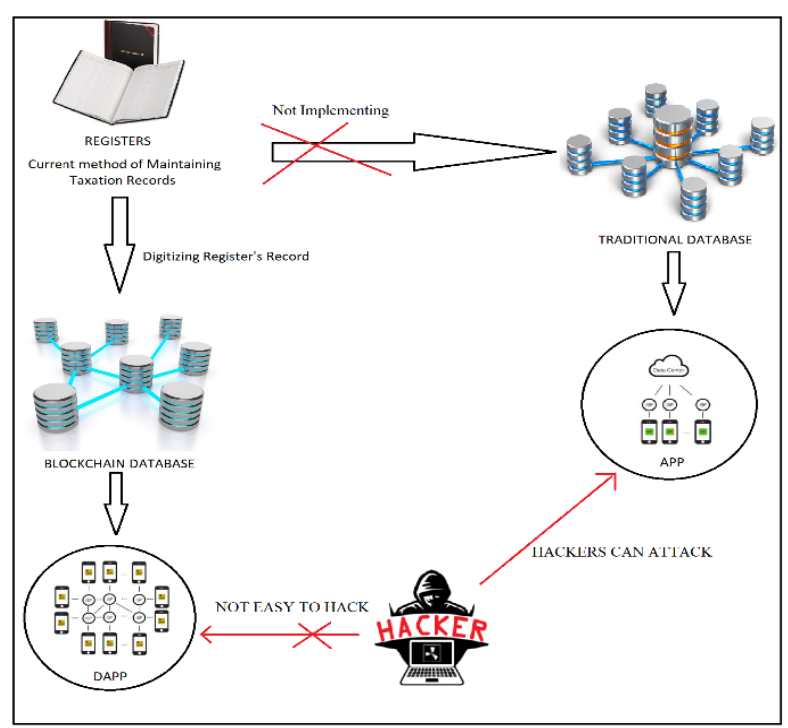

Figure 4 Reason for using DAPP. 
Hacking is relatively tougher in DAPP because of the type of cryptography techniques used in it. As in our system, law is involved and government is involved, so using simple APP for this type of system is not an improvement in system, only digitization is not enough as shown in Figure 4. Security and immutability of data is the most important key features of DAPP which are required for our system [13].

\subsection{Why Permissioned Blockchain Instead of Permission less?}

This type of blockchain is used by specific entities or individuals for the purpose of having private blockchain. As in our system government officials and tax laws are in focus. Permissioned blockchain includes strong authentication process for entering into it and for participating in consensus. It always requires some permission to join into it.

We want that only tax officers and tax inspectors can join into our blockchain network, no other entity should be entered, as our tax records are highly sensitive data which should not be exposed to any other entity and must be kept privately [14].

\section{CONCLUSION}

In this paper, we have proposed a solution for the problem which is being face by taxation department in Pakistan. The records that are being maintained on copy registers are to be digitized and database should be blockchain distributed ledger database, so that frauds must be stopped and logs for each action are generated of senior tax officers and tax practitioners.

Senior tax officers should also be monitored by any way that they are monitoring the junior staff on the system regularly and in the right way or not. This system should be implemented in every government sector so that there is reduce in law breakers and bribe takers.

\section{REFERENCES}

[1] "Tax categories and their rates in Pakistan", 2021 [Online]. Available: https://www.fbr.gov.pk/.

[2] Piracha, M. M., \& Moore, M. (2015). Understanding low-level state capacity: Property tax collection in pakistan.

[3] "Taxation System in Pakistan", 2021 [Online]. Available: https://www.slideshare.net/osmadni/ pakistan-taxation-system-law-presentation

[4] Piracha, M. M. (2016). Sub-national Government Taxation: Case of Property Taxes in Punjab, Pakistan (Doctoral dissertation, University of Sussex).
[5] "Fbr current way of managing records", 2020 [Online]. Available: http://www.excisepunjab.gov.pk/Interview

[6] H., Chen, Zheng, Z., Wang, H Xie, S. \& Dai, X. (2017, June). An overview of blockchain technology: Architecture, consensus, and future trends. In 2017 IEEE international congress on big data (BigData congress) (pp. 557-564). IEEE.

[7] Hassan, B., Farooq, M. S., Abid, A., \& Sabir, N. (2016). Pakistan Sign Language: computer vision analysis \& recommendations. VFAST Transactions on Software Engineering, 4(1), 1-6.

[8] Setyowati, M. S., Utami, N. D., Saragih, A. H., \& Hendrawan, A. (2020). Blockchain Technology Application for Value-Added Tax Systems. Journal of Open Innovation: Technology, Market, and Complexity, 6(4), 156.

[9] Vistro, D. M., Rehman, A. U., Farooq, M. S., Abid, A., \& Idrees, M. (2020). A LITERATURE REVIEW ON SECURITY ISSUES IN CLOUD COMPUTING: OPPORTUNITIES AND CHALLENGES. Journal of Critical Reviews, 7(10), 1446-1455.

[10] Aziz, O., Farooq, M. S., Abid, A., Saher, R., \& Aslam, N. (2020). Research trends in enterprise service bus (ESB) applications: a systematic mapping study. IEEE Access, 8, 31180-31197.

[11] Truby, J. (2018). Bitcoin: Law and policy choices for reducing the energy consumption of Blockchain digital currencies. Energy research \& social science, 44, 399-410.

[12] Hou, H (2018). The application of blockchain technology in E-government in China. Computer Communication and Networks (pp. 1-4). IEEE.

[13] Farid, S., \& Rehman, A. U. (2018). Enhancement in Quality of Services Using Integrated Services in 4G Cellular Network. Technical Journal, 23(03), 82-93.

[14] Rennock, M. J., Butcher, J. R., \& Cohn, A. (2018). Blockchain and regulatory investigations. The Journal, 1, 35-45.

[15] Parameshachari, B.D., Panduranga, H.T. and liberata Ullo, S., 2020, September. Analysis and computation of encryption technique to enhance security of medical images. In IOP Conference Series: Materials Science and Engineering (Vol. 925, No. 1, p. 012028). IOP Publishing.

[16] Kiran, P., Parameshachari, B.D., Yashwanth, J. and Bharath, K.N., 2021. Offline Signature Recognition Using Image Processing Techniques and Back 
Propagation Neuron Network System. SN Computer Science, 2(3), pp.1-8.

[17] Sujan, L.J.L., Telagadi, V.D., Raghavendra, C.G., Srujan, B.M.J., Prasad, R.V., Parameshachari, B.D. and Hemalatha, K.L., 2021. Joint Reduction of Sidelobe and PMEPR in Multicarrier Radar Signal. In Cognitive Informatics and Soft Computing (pp. 457-464). Springer, Singapore.

[18] Hu, L., Nguyen, N.T., Tao, W., Leu, M.C., Liu, X.F., Shahriar, M.R. and Al Sunny, S.N., 2018. Modeling of cloud-based digital twins for smart manufacturing with MT connect. Procedia manufacturing, 26, pp.1193-1203.

[19] Nguyen, T.N., Liu, B.H., Nguyen, N.P. and Chou, J.T., 2020, June. Cyber security of smart grid: attacks and defenses. In ICC 2020-2020 IEEE International Conference on Communications (ICC) (pp. 1-6). IEEE.

[20] Nguyen, N.T., Liu, B.H. and Weng, H.Z., 2018, May. A distributed algorithm: Minimum-latency collision-avoidance multiple-data-aggregation scheduling in multi-channel WSNs. In 2018 IEEE International Conference on Communications (ICC) (pp. 1-6). IEEE.

[21] Subramani, P., Rajendran, G.B., Sengupta, J., Pérez de Prado, R. and Divakarachari, P.B., 2020. A block bi-diagonalization-based pre-coding for indoor multiple-input-multiple-output-visible light communication system. Energies, 13(13), p.3466.

[22] Shivappriya, S.N., Karthikeyan, S., Prabu, S., Pérez de Prado, R. and Parameshachari, B.D., 2020. A modified ABC-SQP-based combined approach for the optimization of a parallel hybrid electric vehicle. Energies, 13(17), p.4529.

[23] L. Tan, N. Shi, K. Yu, M. Aloqaily, Y. Jararweh, “A Blockchain-Empowered Access Control Framework for Smart Devices in Green Internet of Things", ACM Transactions on Internet Technology, vol. 21, no. 3, pp. 1-20, 2021,https://doi.org/10.1145/3433542.

[24] K. Yu, L. Tan, X. Shang, J. Huang, G. Srivastava and P. Chatterjee, "Efficient and Privacy-Preserving Medical Research Support Platform Against COVID-19: A Blockchain-Based Approach", IEEE Consumer Electronics Magazine, doi: 10.1109/MCE.2020.3035520.

[25] C. Feng et al., "Efficient and Secure Data Sharing for 5G Flying Drones: A Blockchain-Enabled Approach," IEEE Network, vol. 35, no. 1, pp. 130137, January/February 2021, doi: 10.1109/MNET.011.2000223.
[26] L. Tan, H. Xiao, K. Yu, M. Aloqaily, Y. Jararweh, "A Blockchain-empowered Crowdsourcing System for 5G-enabled Smart Cities", Computer Standards \&

Interfaces 